\title{
ON THE PERFORMANCE BENEFITS OF WDM AND WAVELENGTH CONVERSION IN PHOTONIC PACKET SWITCHING NODES
}

\author{
Moisés R. N. Ribeiro ${ }^{1.2}$ and Mike J. O'Mahony ${ }^{2}$

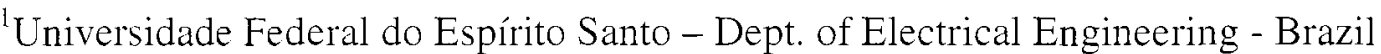 \\ ${ }^{2}$ University of Essex- Dept. of Electronic Systems Engineering- U.K. \\ moises@ele.ufes.br, mikej@essex.ac.uk
}

\begin{abstract}
This paper presents an analytical study concerning the performance of photonic packet switching nodes under both uncorrelated and correlated traffic. An exact and clear solution is proposed to nodes under on-orf traffic. The exploitation of the wavelength domain through WDM and wavelength conversion is investigated, showing that it might simultaneously improve the node capacity. reduce the sensitivity to variations in traffic features. and decrease the buffer depth required to reach a given performance.
\end{abstract}

Resumo - Este artigo apresenta um estudo analítico sobre a performance de nós de comutação fotônica de pacotes submetidos a duas modalidades de tráfego: descorrelacionado e correlacionado. Uma solução exata e explicita é apresentada para nós sob tráfego correlacionado do tipo "on-off". A exploração do domínio dos comprimentos de onda pelo uso de WDM e conversão de comprimentos de onda é investigada, mostrando que isto poderia, simultaneamente, aumentar a capacidade do nó de comutação, reduzir a sensitividade a variações nas características do tráfego além de diminuir a profundidade do buffer para atender a um determinado desempenho.

Keywords: optical networks, photonic switching. packet switching, optical buffering, correlated traffic.

\section{INTRODUCTION}

In a digital network supporting integrated services, the diversity of data rates and its variability in time naturally lead to architectures based on the packet switching concept. It is well known that packet switching better utilises link resources and it is able, at the same time. to accommodate different services, even when each of them has particular requirements. The Asynchronous Transfer Mode (ATM) is an example of packet switching use in order to provide flexible and reliable broadband services. Moreover, the recent growth of new Internet-based services, i.e. voice and video, has shown that the Internet Protocol (IP) may be a cost-effective platform to be used over ATM or even directly on the optical network [1].

As far as the physical layer is concerned. transmission technology in broadband networks will based on optical fibres since there is no transmission medium with higher bandwidth-distance product. The "optical ether" offers bandwidth as broad as $30 \mathrm{THz}$ in addition to low dispersion. Presently, optical amplifiers can be used to counteract fibre attenuation. eliminating the need for electronic regeneration resulting in a bit-rate transparent transmission media.

On the other hand. the requirements of data buffering and processing of packet headers have resulted in electronic solutions for most of the existing fast packet switching nodes. Although optical links can be transparent for bit rate increases, further upgrades, in the near future, are going to be limited by electronic processing in the nodes. Therefore, the advantages of packet switching can meet the full optical fibre capacity through the photonic packet switching technology.

This paper is focused on the benefits of exploiting the wavelength domain in order to improve capacity and performance of photonic switches. In Section 2 the photonic-switched layer is introduced, including a description of the node through functional blocks. The traffic modelling and the performance evaluation in terms of packet loss ratio are shown in Section 3. The packet delay issue is considered in Section 4. followed by an analysis of performance sensitivity in Section 5. Finally, conclusions are drawn summarising the benefits found by using WDM and wavelength conversion in photonic switching nodes.

\section{PHOTONIC-SWITCHED LAYER OVER ALL-OPTICAL NETWORKS}

Optical fibres are widespread in networks interconnecting Synchronous Digital Hierarchy (SDH) nodes as the present strategy to support the foreseen traffic requirements. In addition, SDH offers the management capability needed to provide broadband and multifaceted services.

However. deployment of Wavelength Division Multiplexing (WDM) is already taking place in order to increase capacity of installed links. Nevertheless, it does not seem to be enough. It has been observed in metropolitan and trunk networks that much of the traffic reaching a network node is addressed to another node, so a significant network advantage can be gained from developing optical systems capable of using coarse optical routing to reduce switching requirements at the nodes [3]. Therefore, optical 
networking based on WDM, optical cross connect (OXC), and optical add-drop multiplexer (OADM) represents a first step towards the all-optical network and also means cost reduction for switching equipment in $\mathrm{SDH}$ backbones.

The so-called all-optical network would be able to provide a transparent transmission media for carrying, at the same time, different protocols (format transparency) and bit rates (data-rate transparency). However, its full potential can not be reached in systems having electronic nodes in the communication path. Moreover. the techniques mentioned above increase network capacity and reliability by a circuitswitched or. if you like, wavelength-switched architecture. Consequently, the adoption of more advanced network solutions in the medium term horizon, as packet switching, may become necessary to add flexibility to the optical network and to reach the required throughput of the forthcoming services.

Once the lower layer provides an optical path (or a set of them), nodes operating optically on a packet-switching basis can be connected together. The introduction of a layer which maintains the bit rate and transfer mode transparency will also be a further step to bridge the granularity gap between high speed wavelength channels and the electrically switched network. A network evolution scenario following the mentioned concepts can be seen in Fig. 1 .

This approach improves the network in terms of bandwidth utilisation and flexibility since it allows the witching of frequently changing high bit rate connections without needing wavelength reconfigurations at the OCXs. There are many challenges posed to transport network designers. However, an optical packet layer can transparently support and transport any service coming from the electric switched layer, thereby offering a potential costcompetitive solution for the future telecommunication network with full upgradability features that can replace the SDH circuit-switched architectural paradigm.

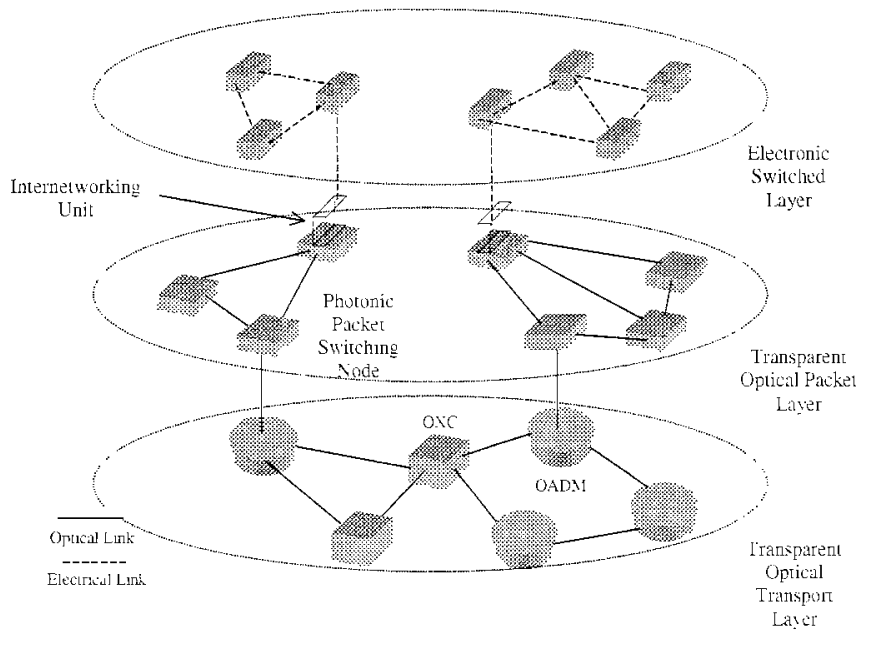

Figure 1. Network architecture with transparent packet layer and OXC - OADM facilities in the transport layer.

Indeed, the unpredictable increase in bandwidth demand and the existence of different packet technologies is the best scenario to use of a transparent packet optical layer. Despite the commercial success of IP and the overwhelming efforts to improve its routing/forwarding abilities and quality of service control, it is unlikely that one technology will dominate in the near future. One should bear in mind that the transport modalities are optimised for the application they serve, e.g. IP for non-real-time services and ATM for real-time and quality ensured deliveries. Accordingly, the presence of internetworking units with the ability of interfacing different packet technologies from electronically switched layers (LANs, MANs or even highbit rate desktop applications) with the optical layer is necessary.

The internetworking units placed at edge switches, shown in Fig.1, are then required to provide a smooth transition between electrical and optical partitions of the network. This interface, illustrated in Fig. 2, is in charge of basic tasks such as: data rate adaptation and convergence, header generation and rewriting, multiplexingdemultiplexing of optical packets, and wavelength conversion [4].

The effects of short and long-term correlation of the incoming electrical packets can also be significantly reduced by traffic shaping in the adaptation unit by using a proper scheduling policy, i.e. the methodology of filling the optical packets, as shown in [5]-[7].

It should be said that, presently, the term all-optical used in photonic packet switching implies that at least the data portion of a packet (payload) remains in optical format from the source to the destination. While the data remains in the optical domain, both fully optical and optoelectronic techniques have being used to process packet routing information contained in the header.

In conclusion, it is not surprising that a significant effort has being made to merge photonic and fast packet switching, since the success achieved by introducing optical technology in the physical layer is also expected by its use in the network level.

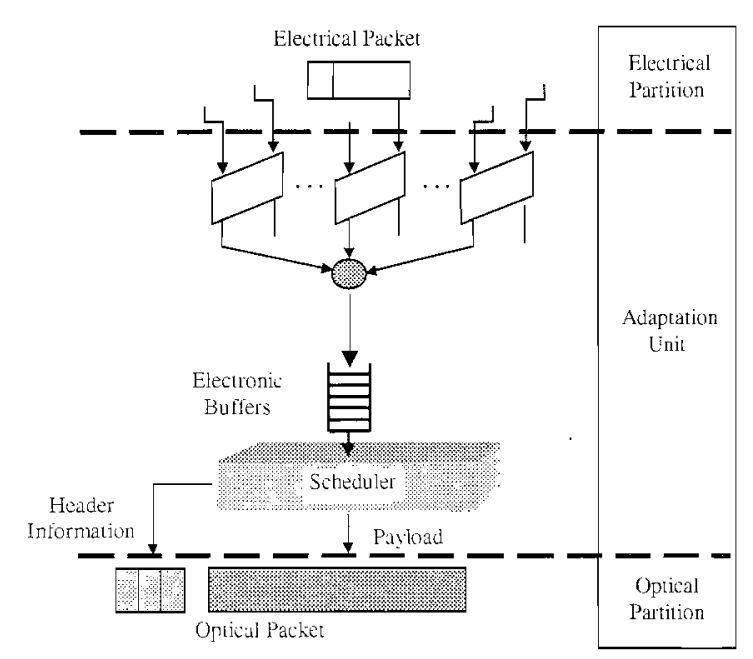

Figure 2. Internetworking Unit. 


\subsection{PHOTONIC SWITCHING NODE}

Although photonic switching tries to follow the footsteps of its successful electronic counter-part. there are fundamental differences between them. One basic distinction is that data in the optical domain can not yet be statically buffered. Buffering is a key issue to keep low packet loss probability :-:ne it is extensively used for contention resolution, i.e. whenever two or more packets are addressed to the same output in a given time slot. On the other hand. there is one more degree of freedom in photonics where schemes using WDM can also be utilised to solve contention.

Fig. 3 shows the basic architecture of a photonic switching node, which utilises Wavelength Division Multiplexing (WDM) and wavelength conversion. At the input individual wavelengths are separated by a demultiplexer. Packet headers are read, and the payloads are optically delayed to allow time for the node control algorithm to set up the path through the switching matrix. In case of contention the node control assigns wavelength conversion and/or buffering. The simplest optical buffering is implemented with fibre-delay. Note that packets pass through a wavelength adaptation block before reaching the output fibre. This block is required whenever more wavelengths are in the node than in the interconnecting network. which depends on the node architecture chosen [10] or the space switching technique utilised [11].

An $\mathrm{NxN}$ node with $\mathrm{n}$ wavelengths per fibre and carrying optical packets with fixed-length (in time) is the case under study. Non-blocking switching is also assumed. In fact. if no blocking is experienced through the spaceswitching matrix photonic node can be studied as being output buffered. The buffering process in packet switching can be implemented at the input, at the output, or even in an shared way by a specific output connected to a buffer that feeds back blocked packets to the input of the space switch. The output configuration shows best performance in terms of cell loss probability and delay without breaking the cell sequence [12]. In this case, however, it should be provided that the switch matrix is $\mathrm{nN}$ times faster (speed-up factor), than the link capacity (packets/s) so that even nN packets. from $\mathrm{nN}$ inputs. can be addressed to a given output in the same time slot. Synchronous operation is considered in this paper, which can be supplied by correct fibre length between nodes or, more realistically, optical synchronisers placed just after the header processor [13][14].

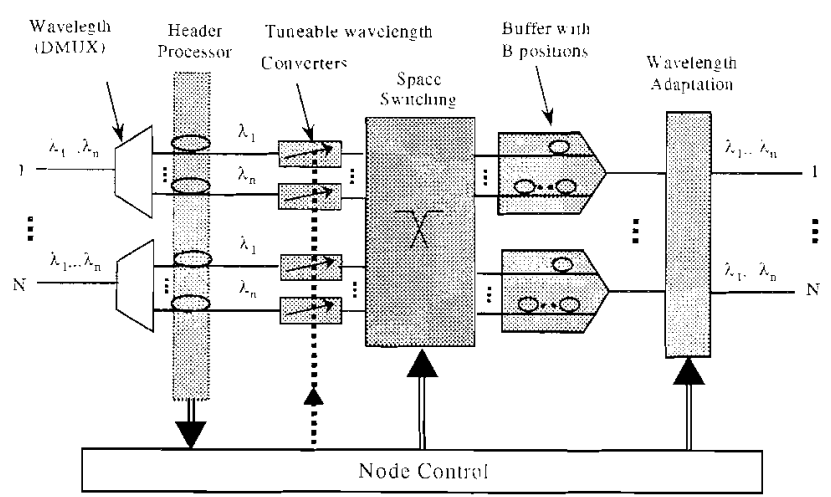

Figure 3. Basic architecture of a photonic switching node with $\mathrm{NxN}$ fibres and $\mathrm{n}$ wavelengths per fibre.
Concerning the buffering system composed of fibredelays. the use of WDM (without wavelength conversion) readily increases the node capacity by $n$ times. For instance, a $4 \times 4$ switch can be turned into a $16 \times 16$ by carrying 4 wavelengths in each fibre instead of only one, provided that the space switching is able to cope with that without blocking packets. Notice that in this case the packets have to leave the node using the same wavelength on which they have arrived. On the other hand, if tuneable wavelength conversion is also used. as shown in Fig. 2, a packet can leave the node using a different wavelength: creating then more alternatives for contention resolution. In doing so, the packet sequence may be broken. but simulations have shown that this effect is negligible for nodes operating with a packet loss probability less than $10^{-5}[10]$.

\section{TRAFFIC MODELLING PERFORMANCE EVALUATION}

Packet loss probability will be studied under both uncorrelated and correlated traffic. This section uses: $\mathrm{N}$ as the number of fibres, $\mathrm{n}$ for number of wavelengths per fibre, while $B$ stands for the buffer depth, and $\rho$ represents the offered load per wavelength channel.

\subsection{UNCORRELATED (RANDOM) TRAFFIC}

The Packet arrival is modelled as a Bernoulli process, then the arrival process to the output buffer follows a binomial distribution (uncorrelated) [10][15]. Equal probability is assumed for the destination of packets, i.e. each output buffer has an offered load equals to the input offered load per wavelength channel divided by the number of outputs. Then the probability of having $\mathrm{k}$ arrivals during a time slot is given by (1). Notice that even $\mathrm{nN}$ packets can be addressed to the same output.

$$
a_{k}=\left(\begin{array}{c}
n N \\
k
\end{array}\right)\left(\frac{\rho}{N}\right)^{k}\left(1-\frac{\rho}{N}\right)^{n N-k}
$$

Since WDM is used, $\mathrm{n}$ packets can be served in the same time slot they arrive, so in order to have one packet buffered, $n+1$ must be arrived. A Markov chain is used to represent the buffer state probability $q_{i}$. The transition probability among states (from $i$ to $j$ ) for this Markov chain is given in (2) where $0 \leq \mathrm{i}, \mathrm{j} \leq \mathrm{B}$

$$
P_{i j}= \begin{cases}\sum_{j=0}^{n-i} a_{i} & 0 \leq i \leq n, j=0 \\ a_{j-i+n}, & 0 \leq i \leq n, 1 \leq j \leq B-1 \\ a_{j-i+n}, & n+1 \leq i \leq B, i-n \leq j \leq B-1 \\ \sum_{l=B-i+n}^{n N} a_{l} & 0 \leq i \leq B, j=B \\ 0 & \text { otherwise }\end{cases}
$$

The steady-state solution of the Markov chain can be found by iteration of (2) with an arbitrary solution, as shown in (3). using $\mathrm{P}_{\mathrm{jj}}$ in a matrix format and $m$ represents the time slot. 


$$
\left[\begin{array}{lll}
\mathrm{q}_{0}^{\mathrm{m}-1} & \cdots & \mathrm{q}_{\mathrm{B}}^{\mathrm{m}-1}
\end{array}\right] \underset{=}{\mathrm{P}}=\left[\begin{array}{c}
\mathrm{q}_{0}^{\mathrm{m}} \\
\vdots \\
\mathrm{q}_{\mathrm{B}}^{\mathrm{m}}
\end{array}\right]^{\mathrm{T}}
$$

From the buffer occupancy probability, the average number of unused wavelength can be calculated as stated in (4).

$$
\rho_{0 \lambda}=\sum_{i=0}^{n} \sum_{j=0}^{n-1} a_{j} q_{i} \cdot[n-(i+j)]
$$

The normalised throughput (NTP) is then found using (5). Finally, the packet loss probability (PLP) calculation is obtained in (6):

$$
\begin{aligned}
& \text { NTP }=\frac{n-\rho_{0 i}}{n} \\
& \text { PLP }=1-\frac{N T P}{\rho}
\end{aligned}
$$

The modelling presented above is. indeed, a queuing system with $\mathrm{n}$ servers. In addition, due to wavelength conversion, $n$ packets can share each buffer position in the same time slot, so that it is equivalent to having $\mathrm{nB}$ buffer positions available in the node. In order to find out the individual effect of multiple servers and buffer sharing on the final performance. the result of a set-up that does not allow buffer sharing is presented in Fig. 4(a).

It can be seen that the contribution of just having multiple servers is small to reduce the packet loss ratio. Much better results, however, are achieved when the buffer sharing is also implemented. Using ten fibre-delays and $\mathrm{n}=4$, for example, the packet loss probability is decreased by 6 orders of magnitude, as can be seen in Fig. 4(b). Therefore, it can be concluded that the main benefit of WDM to the packet loss probability arises from the buffer sharing within the node.

The evaluation of buffer depth reduction versus wavelength channels for packet loss ratio lower than $10^{-10}$ is presented in Fig. 5. Results for larger values of $\mathrm{N}$ are very similar to those found for $16 \times 16$.

As expected. the required buffer depth for a given PLR approximately decreases by $1 / \mathrm{n}$, due to the multiplication by $\mathrm{n}$ of buffer positions.

\subsection{CORRELATED (BURSTY) TRAFFIC}

The results obtained so far using random traffic modelling are possibly optimistic because the packet arrival sequence is considered uncorrelated. A more elaborated approach should be used to take into account effects of traffic correlation on the node design since most of the actual traffic sources produce temporal correlation among packets.

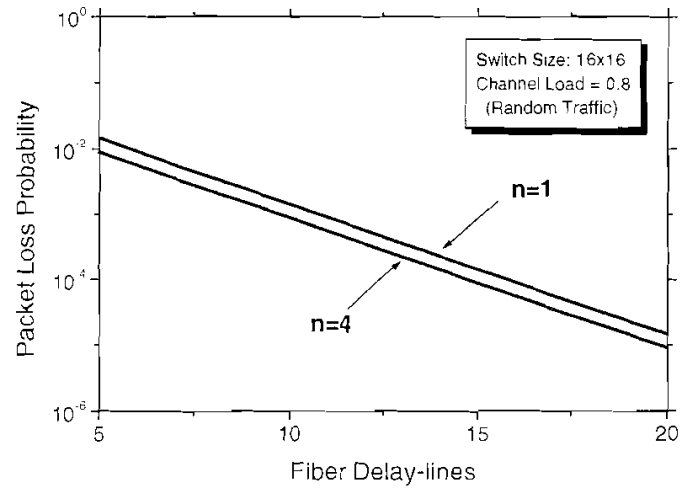

(a)

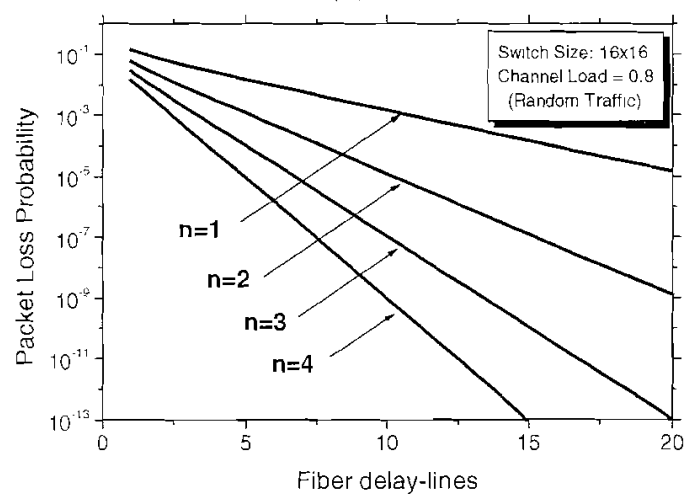

(b)

Figure 4. Packet Loss Probability versus number of fibredelays (buffers) for nodes using WDM. (a) Without buffer sharing. (b) With buffer sharing.

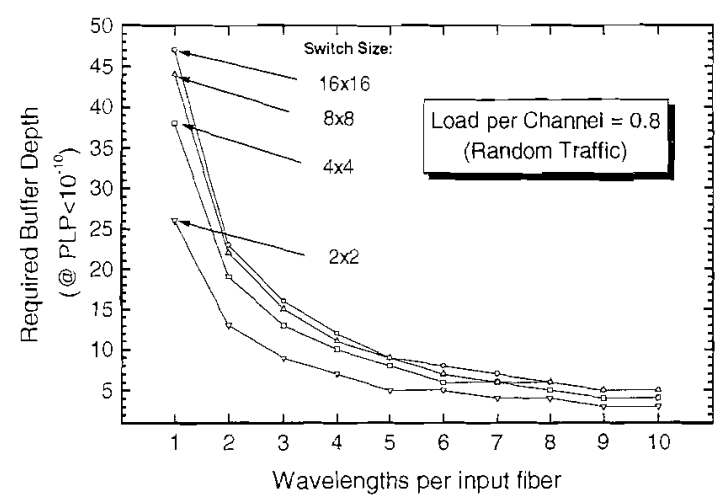

Figure 5. Required buffer depth, for packet loss ratio less than $10^{-10}$. versus number of wavelength per fibre. 
In the correlated traffic model used in this paper, each identical input source is taken as following the Markov Modulated Bernoulli Process [17], as shown in Fig.6.

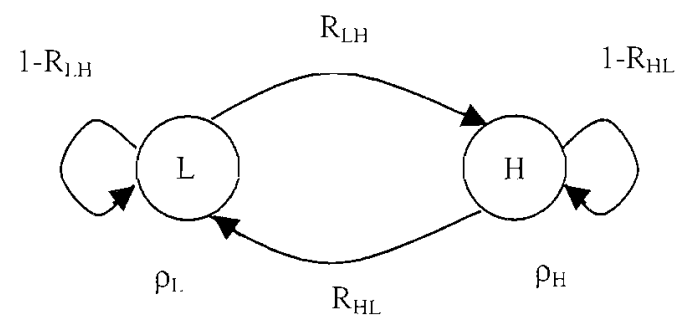

Figure 6. Traffic Source modelled as Markov Modulated Bernoulli Process.

There are two possible states for each source to be concerning the traffic generated: $L$ (low) and $H$ (high). producing an offered load $\rho_{\mathrm{L}}$ and $\rho_{\mathrm{H}}$, respectively. RLH and RHL represent the transition probabilities between states. from low to high and high to low respectively.

The number of inputs in state $\mathrm{H}$ addressed to the outlet under observation is taken as random and modelled by the binomial distribution. It is possible to find between 0 and $\mathrm{nN}$ sources in state $\mathrm{H}$ and the transition probability is given by (7).

$$
\begin{aligned}
& Q_{x . y}= \sum_{k=\max (0 \cdot x-y)}^{\min (x, n \cdot N-y)} A_{L}[k \backslash x] \cdot A_{H}[k-x+y \backslash n \cdot N-x] \\
& A_{L}[k \backslash x]=\left(\begin{array}{l}
x \\
k
\end{array}\right) R_{H L}^{k}\left(1-R_{H L}\right)^{i-k} \\
& A_{H}[k-x+y \backslash n N-x]=\left(\begin{array}{c}
n N-x \\
k-x+y
\end{array}\right) R_{L H}^{k-x+y}\left(1-R_{L H}\right)^{n N-k-y}
\end{aligned}
$$

The Markov chain representing the buffer state, in this ase, should also hold the number of inputs in $\mathrm{H}$ state, zomprising $(\mathrm{nN}+1) \times(\mathrm{B}+1)$ elements and the iteration to find out the steady-state is performed as shown in (8).

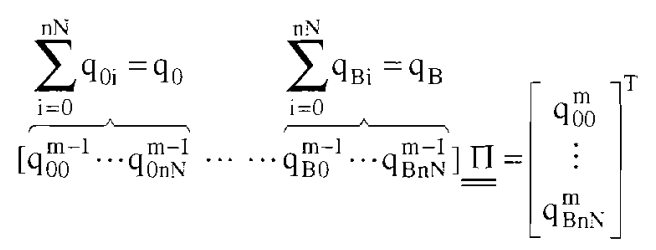

Where $\underline{\Pi}$ represents the transition matrix berween buffers states, which has $(\mathrm{B}+1) \times(\mathrm{B}+1)$ elements as shown in (9). However, each element of this matrix is a sub-matrix with $(\mathrm{nN}+1) \times(\mathrm{nN}+1)$ elements, which takes into account the transition among source states, as can be seen in (10):

$$
\underline{\underline{\Pi}}=\left[\begin{array}{ccc}
\underline{\pi_{0.0}} & \cdots & \underline{\pi_{0 . \mathrm{B}}} \\
\bar{\vdots} & \ddots & \bar{\vdots} \\
\underline{\underline{\pi_{\mathrm{B} .0}}} & \cdots & \underline{\underline{\pi_{\mathrm{B} . \mathrm{B}}}}
\end{array}\right]
$$

$$
\stackrel{\underline{\pi_{i . j}}}{=}\left[\begin{array}{ccc}
P_{i . j}^{\{0\}} Q_{0.0} & \cdots & P_{i . j}^{\{0\}} Q_{0 . n N} \\
\vdots & \ddots & \vdots \\
P_{i . j}^{\{n N\}} Q_{\Omega .0} & \cdots & P_{i . j}^{\{n N\}} Q_{n N . n N}
\end{array}\right]
$$

Notice that $P_{i . j}^{\{c\}}$ is the same shown in (2) but the arrival probability is given by (11), instead of (1), where $c$ is the number of sources in $\mathrm{H}$ state.

$$
a_{r}^{i c j}=\sum_{k=1}^{r}\left(\begin{array}{l}
c \\
k
\end{array}\right)\left(\rho_{H}\right)^{k}\left(1-\rho_{H}\right)^{c-k}\left(\begin{array}{c}
n N-c \\
r-k
\end{array}\right)\left(\rho_{L_{H}}\right)^{1-k}\left(1-\rho_{L}\right)^{n N-c-r+k}
$$

The results presented in this paper are for traffic sources having on-off behaviour, as found in [18] but using an exact solution instead. This particular case of Markov modulated Bernoulli process is called interrupted Poisson process. During the $H$ period packets are emitted with $\rho_{H}=$ 1 and for $L$ period with $\rho_{L}=0$, in other words, continuous emission of packets for $\mathrm{H}$ state and no packet emission in the $\mathrm{L}$ period. For this case, it is easy to find a connection of the source model parameters with the burstiness and the offered load. Furthermore, the steady-state solution found by (8) can be applied to (4)-(6) in order to work out the packet loss probability.

Applying local balance boundary to the source model shown in Fig. 6. one can find that:

$$
\operatorname{PH}=\operatorname{Prob}[\text { state }=H]=\frac{R_{L H}}{R_{L H}+R_{H L}}
$$

If packets are exclusively released in the state $\mathrm{H}$ and the incoming traffic is considered equally distributed among the output ports, then

$$
\begin{aligned}
& \mathrm{R}_{\mathrm{LH}}=\frac{\frac{\rho}{\mathrm{N}} \mathrm{R}_{\mathrm{HL}}}{1-\frac{\rho}{\mathrm{N}}} . \\
& \text { where: } \mathrm{R}_{\mathrm{HL}}=1 / \beta
\end{aligned}
$$

The mean burst length, or burstiness $(\beta)$, represents the mean number of packet arriving in sequence to a given output buffer. The effect of traffic correlation on the node performance can be realised comparing the variation of packet loss probability versus offered load and burstiness, as shown in Fig. 7 for nodes using a single wavelength per fibre. 


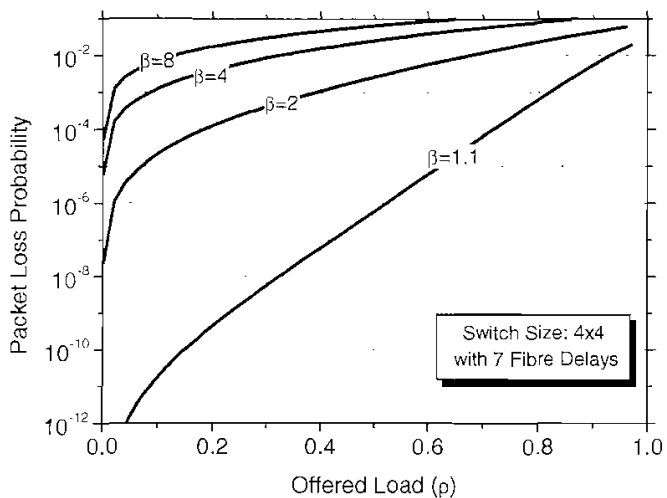

(a)

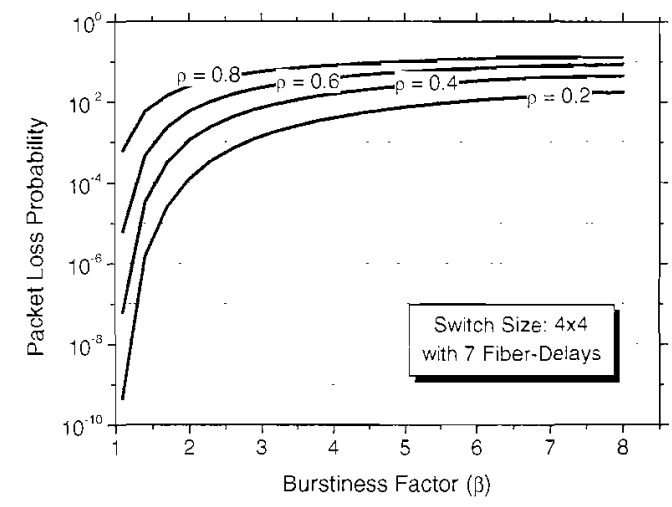

(b)

Figure 7. Effect of correlated traffic in the packet loss performance. (a) PLP versus offered load using burstiness as the parameter. (b) PLP versus burstiness using the offered load as the parameter.

It is clear that the burstiness factor has a massive influence on the node performance, little variation of burstiness can take the node performance to unacceptable values of packet loss probability. For instance, in Fig. 7(a) for an offered load of 0.2 and burstiness of 1.1 the packet loss is less than $10^{-9}$. Despite keeping the same offered load, the PLP is increased to $10^{-4}$ as soon as two packets on average arrive in sequence. Comparing Figs. 7(a) and (b), one can see that the performance seems to be more sensitive to burstiness variation than to offered load changes.

The benefits of WDM and wavelength conversion in a node under correlated traffic now will be investigated. In Fig. 8 the case presented in Fig. 7(a) using $\beta=4$ is revisited to show the improvements brought by the exploitation of the wavelength domain.

Notice that the performance shown in Fig. 8 is quite improved when more than one wavelength is used. roughly following $\beta / n$, i.e. the performance for $n=2$ and $n=4$ in Fig. 8 are close to the PLP presented in Fig. 7(a) for $\beta=2$ and $\beta=1.1$ respectively.

As in the case of random traffic, improvements can be seen on the buffer depth requirement as shown in Fig. 9. The buffer size might be reduced from 86 to 21 if 4 wavelength are used to reach a PLP of $10^{-10}$.

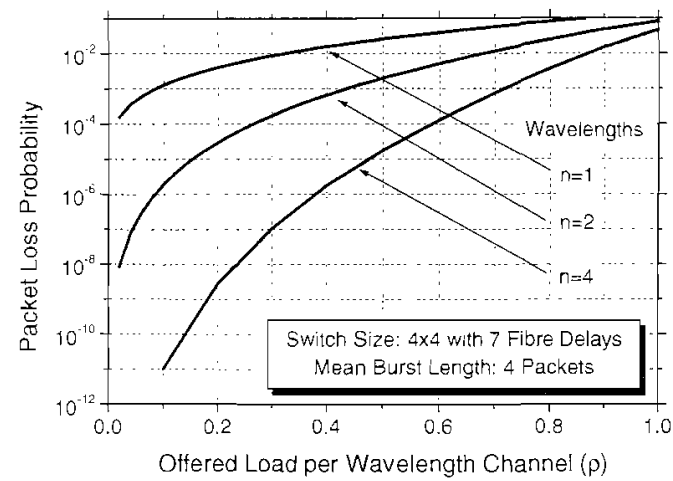

Figure 8. Packet Loss Probability versus offered load for a node with WDM under correlated traffic.

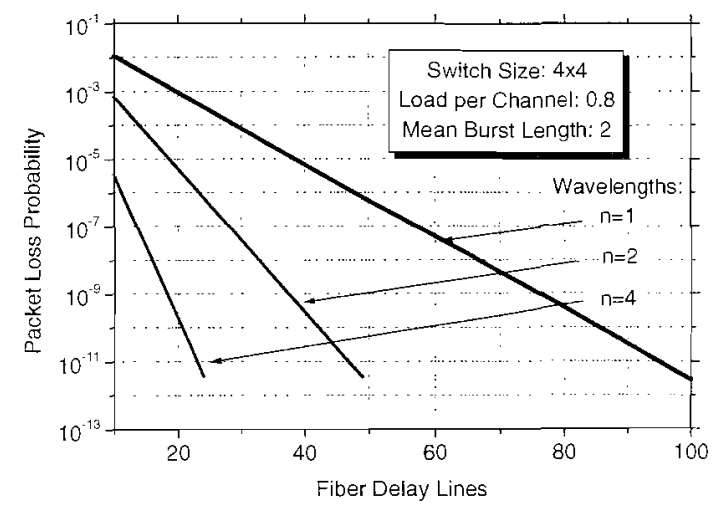

Figure 9. Packet Loss Probability versus number of fibredelays (buffers) for nodes under correlated traffic and using WDM and wavelength conversion.

\section{THE PACKET DELAY ISSUE}

In packet-switched networks there are two basic metrics for the assessment of quality of service (QoS), namely, packet loss probability and mean packet delay. This section investigates the delay introduced in switching nodes due to the queuing system. Both cases of nodes under random and correlated traffic will be studied.

Once the steady-state probability of buffer occupancy is found, the mean waiting delay suffered by packets due to the output buffering is straightforward through the wellknown Little's law from queuing theory [12][15]. Fig. 10(a) shows the variation of mean waiting time versus offered load for various buffer depths for uncorrelated traffic. In addition, a bound is stated for an infinite switch with infinite buffer, which corresponds to a $\mathrm{M} / \mathrm{D} / 1$ queuing system. It is clear that the mean delay for a large switch converges to the bound curve for uncorrelated traffic. Nevertheless, when traffic has temporal correlation features the picture is changed, as shown in Fig. 10(b) and (c). 


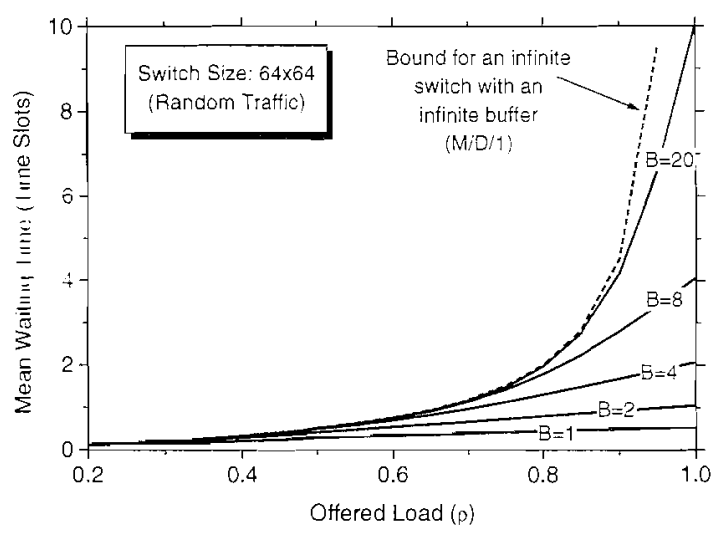

(a)

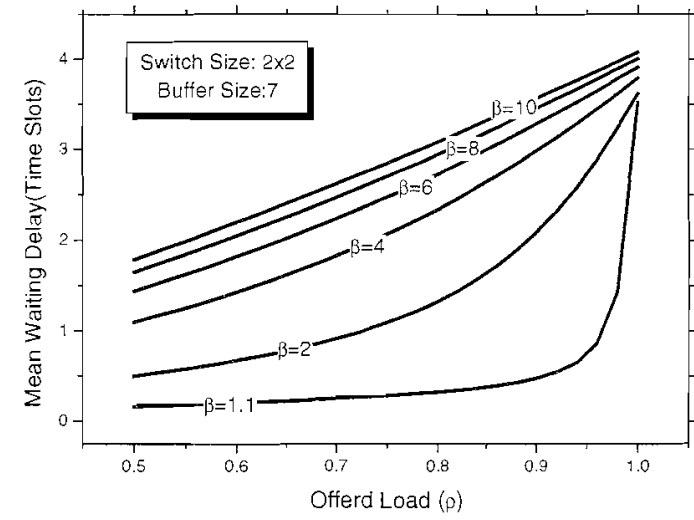

(b)

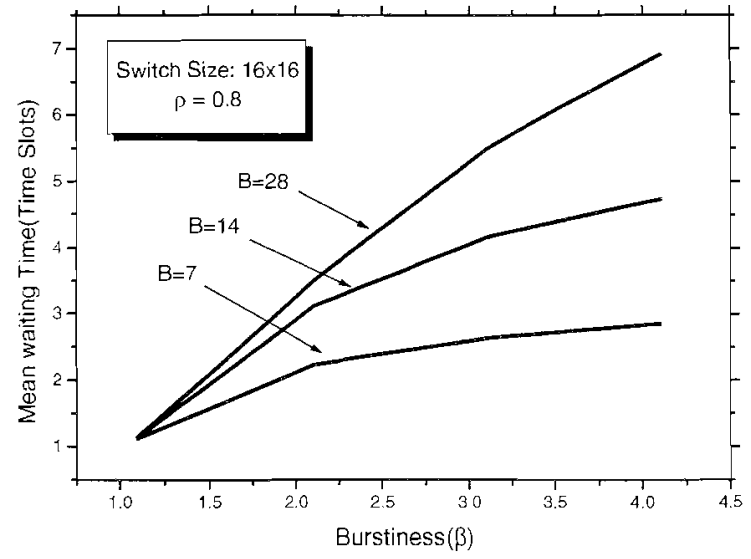

(c)

Figure 10. Assessment of delay in photonic switched node. (a) Uncorrelated traffic. (b) Effect of correlation in delay characteristics. (c) Effects of buffer depth and burstiness variation.

In Fig. 10(b) it is shown that the exponential behaviour for uncorrelated traffic is replaced by a linear variation against offered as the burstiness factor increases. This effect can be understood if the delay for uncorrelated traffic with few buffers, i.e. 1 to 4 , in Fig. 3(a) is carefully analysed. In this case, the buffer occupancy probability is high, which also means high loss probability, then it is likely that any further arrival will be lost instead of being added to the amount of packets stored in the buffer to increase the mean delay value. The verification of this principle can be seen in Fig 3(c), where the delay saturation versus burstiness was found to be dependent on the buffer depth.
In order to assess the relationship between packet delay and packet loss, the case studied in Fig.7 is revisited and results for mean delay are presented in Fig.11. Comparing both performances, it is evident that for nodes designed to allow packet loss probabilities as low as $10^{-10}$ the mean delay introduced by this node must be negligible since it remains under one time slot. As a matter of fact. the total buffer depth (number of fibre-delays) will impose the upper bound for delay in output-buffered nodes.

Although the use of WDM wavelength conversion allows multiplication of buffer capacity, the upper bound does not change since this multiplication is based on the sharing of a physical buffer. Therefore. delay may not be a critical issue since the node has to work with shallow buffering systems. When a large physical buffer depth is to be used the associated space switching required for addressing the buffers results in increased cost and crosstalk effects

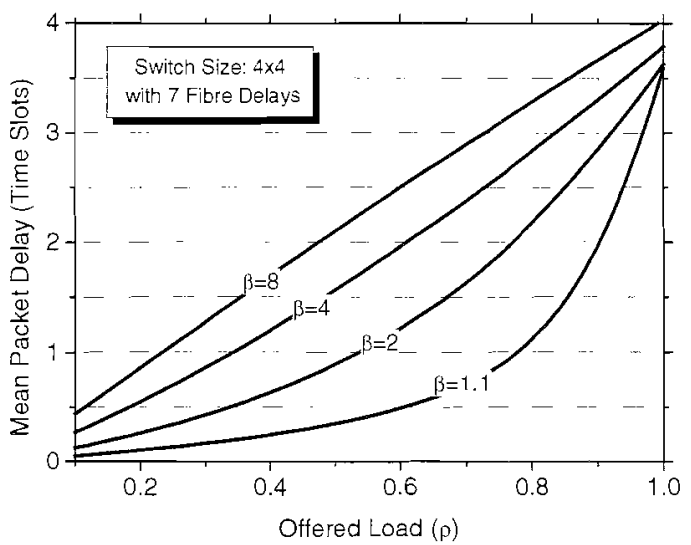

(a)

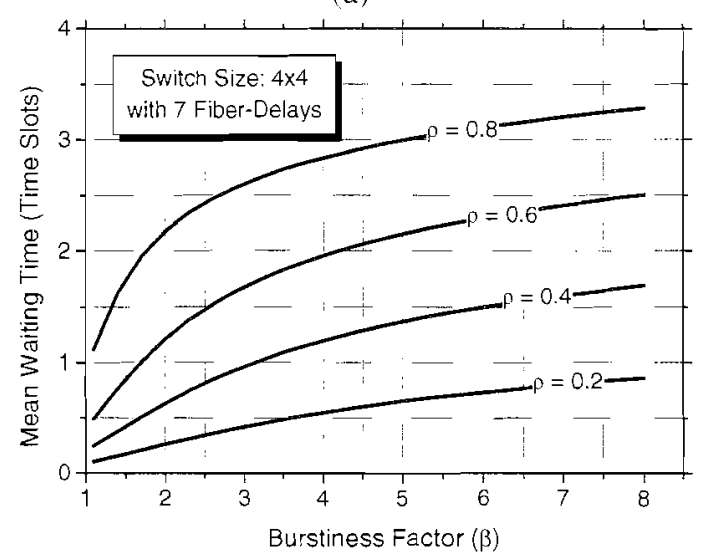

(b)

Figure 11. Packet mean delay. (a) using burstiness as the parameter. (b) using offered load as the parameter.

\section{PERFORMANCE SENSIVITY}

With the purpose of investigating the robustness of improvements brought by WDM and wavelength conversion for nodes under correlated traffic, Fig. 12 presents the sensitivity in terms of acceptable load versus burstiness admitted for packet loss probability of $10^{-10}$ and $10^{-7}$. 


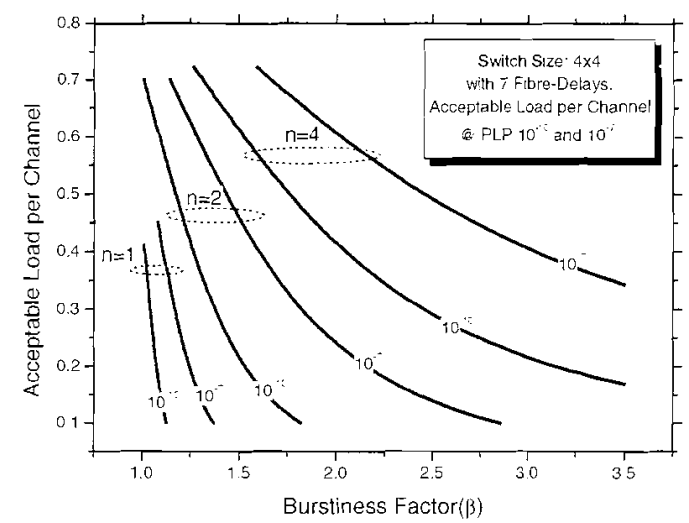

Figure 12. Performance sensitivity for nodes under bursty traffic. Acceptable load versus burstiness factor admitted for packet loss ratio of $10^{-10}$ and $10^{-7}$.

If only one wavelength is in use, the curves for PLP $10^{-10}$ and $10^{-7}$ are quite steep and close to each other, indicating high sensitivity to burstiness variation. However, as more wavelengths are introduced. the accepted load and burstiness are increased and those curves become smoother and more separated, showing that the node can cope with variations in the features of the traffic without severely degrading the packet loss performance. This improvement in tolerance can be explained by the fact that before occupying a new buffer position, the incoming packets can be transmitted using another wavelength or can share a previous occupied buffer position.

\section{CONCLUSION}

The improvements achieved by exploiting the wavelength domain in photonic packet switching were demonstrated through analytical models considering both random and correlated (on-off) traffic. WDM and wavelength conversion enable switch capacity multiplication, reduction in buffer depth, and improved robustness to variation in the traffic features. It has been shown that buffer sharing, enabled by wavelength conversion. is the responsible for most of those improvements. The performance of mean packet delay was also investigated showing that this topic is not relevant for output buffered photonic nodes operating with low packet loss probability.

The introduction of packet priority and buffer management was investigated in other works [20][2]] aiming at best utilisation of node resources (buffers and wavelengths) by taking advantage of different requirements of the incoming packets from the electronic partition.

One can conclude that WDM and wavelength conversion only bring benefits to the node performance. However, their influence on the physical layer should be also analysed to assess the penalty that they might introduce.

\section{REFERENCES}

[1] P. Lagasse et al "Roadmap toward the optical communication age- a European view by HORIZON project and the ACTS photonic Domain". HORIZON-ACTS report.
Nov. 1999. Available at the following URL: www.intec.rug.ac.be/Research/Projects/horizon/welcome.html

[2] G. Prati (editor) "Photonics networks", Springer, 1997.

[3] D. W. Smith (editor) "Optical network technology", BT series, Chapman\&Hall, 1995.

[4] F. Callegati M. Casoti. and C. Rafaelli, "Packet optical network for high-speed TCP-IP backbones" IEEE Comm. Magazine, pp. 124-129, Jan. 1999.

[5] M. Calisti. F. Callegati, "Traffic models for an optical transparent packet network", NOC'97. European Conference on Network and Optical Communications, Antwerp. Belgium. June 1997.

[6] F. Callegati and C. Raffaelli, "End-to-end performance of an optical transparent packet network", IEEE ICC98, Atlanta. June 1998.

[7] P. Gambini et al, "Transparent optical packet switching: network architecture and demonstrator in the KEOPS project", J. on Selected Areas in Comm., vol. 16, pp.12451259. Sep. 1998.

[8] A. Jajszczyk et al, "Photonic Fast Packet Switching", IEEE, Communication Magazine. February 1993.

[9] D. J. Blumenthal et al,"Photonic packet switches: architecture and experimental implementations" Proceedings of IEEE, Vol 82, November 1994.

[10] S. L. Danielsen et al, "WDM Packet Switch Architectures and Analysis of the Influence of Tunable Wavelength Converters on the Performance", IEEE, Journal of Lightwave Tech. Vol. 15, pp 219-226, February 1997

[11] K. M. Guild and M. J. OMahony, "Routing and Buffering architecture in all-optical networks", Electronic Letters, vol. 35. n. 02, Jan. 1999.

[12] M. Hluchyj et al. "Queuing in High-performance Packet Switching", IEEE. Journal of Selec. Areas in Comm. Vol. 6. pp 1587-1589, December 1988.

[13] C. Guillemont et al "Transparent optical packet switching: the European ACTS KEOPS project approach", IEEE. Journal of Lightwave Tech. Vol. 16, pp $2117-2132$, December 1998.

[14] D. Chiaroti et al, "Physical and logical validation of a network based on all-optical packet switching system", IEEE, Journal of Lightwave Tech. Vol. 16, pp 2117-2132, December 1998.

[15] T. G. Robertazzi "Computer Networks and SystemQueuing Theory and Performance Evaluation", 2nd edition, Springer-Verlag, 1994.

[16] T. G. Ghanbari et al. "Principles of Performance Engineering for Telecommunication and Information Systems". IEE Press, 1997.

[17] A. Borella et al, "Statistical Multiplexing of Random Process in Packet Switching Networks", IEE, Proc. Commun. Vol. 143. No 5, pp. 325-334, Oct. 1996.

[18] S. L. Danielsen el al, "Analysis of a WDM Packet Switch with Improved Performance Under Bursty Traffic Conditions Due to Tunable Wavelength Converters", IEEE, Journal of Lightwave Tech. Vol. 16, pp 729-735, May 1998. [19] S. L. Danielsen et al, "Wavelength Conversion in optical Packet Switching", IEEE. Journal of Lightwave Tech. Vol. 16, pp 2117-2132, December 1998.

[20] M. R. N. Ribeiro and M. J. OMahony, "On prioritization and optimal buffer management in photonic packet switching nodes", OFC2000, paper Thu U2, Mar. 2000 . 
[21] M. R. N. Ribeiro and M. J. OMahony, "Improvements on performance of photonic packet switching nodes by priority assignment and buffer sharing", ICC'2000, paper S33.3, Jun. 2000.

Moisés R. N. Ribeiro received his first degree in Electrical Engineering from the Instituto Nacional de Telecomunicacações (INATEL) in 1992 and the Master degree also in Electrical Engineering from the Universidade Esladual de Campinas (UNICAMP) in 1996. Since 1995 he is with the Departamento de Engenharia Elétrica at the Universidade Federal do Espírito Santo (UFES). Presently, he is a Ph.D. sludent at the University of Essex, being funded by $\mathrm{CNPq}$. His research interests are related to performance evaluation of optical systems and networks.
Mike J. O'Mahony received his Ph.D. degree in 1977 from the University of Essex, for research into digita transmission systems. He then joined BT Laboratories reaching the position of Head of Inland Systems Section, responsible for research into long haul optical systems and networks. In 1991 he joined the Department of Electronic Systems Engineering at the University of Essex as Professor of Communications. He is the author of over 200 papers relating to optical communications. Prof. O'Mahony has been recognised as one of the pioneers of the application of wave division multiplexing (WDM) to optical networking and is active with the European Community in photonic networking concentration programmes (ACTS and IST). Lately, he is also involved with a start-up compan! (ILOTRON) which aims to launch the world's first truly alloptical core network router for telecommunications networks. 